\title{
Habilidades Sociais, Comportamentos e Desempenho Acadêmico em Escolares antes e após Intervenção
}

\author{
Luciana Carla dos Santos Elias - Universidade de São Paulo, Ribeirão Preto, Brasil \\ Márcia Viana Amaral - Universidade de São Paulo, Ribeirão Preto, Brasil
}

\begin{abstract}
Resumo
Habilidades sociais (HS) são reconhecidas como fator de proteção no desenvolvimento humano. Objetivou-se avaliar HS, problemas de comportamento e desempenho acadêmico em crianças antes e após treino de HS. Participaram 54 crianças (27 grupo intervenção e 27 grupo comparação), matriculadas no $5^{\circ}$ ano do ensino fundamental, junto à rede municipal de ensino de uma cidade do interior paulista e duas professoras. O treino foi conduzido pela professora de GI em sala de aula e durou quatro meses. Os participantes de GC não receberam intervenção. As crianças foram avaliadas quanto às HS, problemas de comportamento e desempenho acadêmico antes e após intervenção. Foram utilizados: Teste de Desempenho Escolar, Sistema de Avaliação de Habilidades Sociais, formulário para professores e o Programa Posso Pensar, que objetiva desenvolver HS. As análises apontaram resultados superiores de GI em diferentes variáveis. O desenvolvimento de programas de intervenção de caráter universal indica possibilidades concretas de trabalho preventivo.

Palavras-chave: habilidades sociais, problemas de comportamento, desempenho acadêmico, crianças, intervenção
\end{abstract}

\section{Social Skills, Behavior and Academic Performance in School before and after Intervention}

\begin{abstract}
Social skills (SS) are recognized as a protective factor in human development. The objective was to evaluate the SS, behavior problems and academic performance in children before and after SS training. 54 children 27 Intervention Group and 27 Comparison Group) participated in the study, enrolled in the 5th year of elementary school, in a municipal education network of the interior city of São Paulo state and two teachers. The training was conducted by IG teacher in the classroom and lasted about four months. The CG participants received no intervention. The children were evaluated for HS, behavior problems and academic performance before and after the intervention. The instruments used were: Academic Performance Test, Evaluation System of Social skills, formulary for teachers and the Program "I can think", that aims to develop SS. The analysis showed superior results of IG in different variables. The development of a universal nature of intervention programs indicates concrete possibilities of preventive work.

Keywords: social skills, behavior problems, academic performance, children, intervention
\end{abstract}

Habilidades Sociales, Comportamientos y Desempeño Académico en Escolares antes y después de la Intervención

\begin{abstract}
Resumen
Las Habilidades Sociales (HS) son reconocidas como un factor protector en el desarrollo humano. El objetivo fue evaluar HS, problemas de comportamiento y desempeño académico en niños antes y después del entrenamiento de HS. Participaron 54 niños (27 del Grupo de Intervención y 27 del Grupo de Comparación), matriculados en el quinto año de la Enseñanza Primaria, de una ciudad del interior de San Pablo y también dos profesores. El entrenamiento se llevó a cabo por la profesora de GI en sala de aula y duró cuatro meses. Los participantes de GC no recibieron intervención. Los niños fueron evaluados en cuanto a las HS, problemas de comportamiento y desarrollo antes y después de la intervención. Los instrumentos utilizados fueron: Test de Desempeño Escolar, Sistema de Evaluación de Habilidades Sociales, formulario para los profesores y el Programa Puedo Pensar, con el objetivo de desarrollar HS. Análisis mostraron resultados superiores de GI en diferentes variables. El desarrollo de programas de intervención de carácter universal indica posibilidades concretas de trabajo preventivo.

Palabras clave: habilidades sociales, problemas de comportamiento, desempeño académico, niños, intervención
\end{abstract}

Os anos do ensino fundamental configuram-se em um período de desenvolvimento chave no qual as habilidades essenciais para o posterior sucesso acadêmico e social são adquiridas e afinadas (McCabe \& Altamura, 2011). Dentre tais habilidades, as habilidades sociais (HS) tornam-se um fator importante para o ajustamento social e sucesso no ambiente escolar (Borges \& Marturano, 2010; Del Prette \& Del Prette, 2003).

Muitas são as definições de HS encontradas na literatura. Tais habilidades dizem respeito a comportamentos necessários a uma relação interpessoal bem-sucedida, conforme parâmetros típicos de cada contexto e cultura (Caballo, 2003; Falcone, 2002; Murta, 2005). De acordo com Caballo (1996, 2010) e Del Prette e Del Prette (2005), esses comportamentos ainda devem colaborar para a resolução de problemas imediatos em uma dada situação e reduzir a probabilidade de ocorrência de problemas futuros, contribuindo para a manutenção de um relacionamento saudável e produtivo com as demais pessoas. 
Portanto, para definir as HS, deve-se levar em consideração tanto o conteúdo quanto as consequências dos comportamentos interpessoais.

Conforme Del Prette e Del Prette (2005), as HS contribuem para a competência social. Para esses autores, a competência social tem um sentido avaliativo e está relacionada à capacidade de articular pensamentos, sentimentos e ações em função de objetivos pessoais e de demandas da situação e da cultura, gerando consequências positivas para o indivíduo e para a sua relação com as demais pessoas.

Segundo Falcão (2014), as HS são mantidas por suas consequências. Dessa forma, quanto mais socialmente habilidoso for o indivíduo, maior a probabilidade de obter reforçadores. Bolsoni-Silva e Carrara (2010) ressaltam que as HS são funcionalmente concorrentes aos problemas de comportamento, ou seja, são as consequências do comportamento que aumentam ou diminuem a probabilidade deste voltar a ocorrer e, quando duas topografias de comportamento possuem a mesma função, eles se tornam concorrentes. Cia e Barham (2009) ressaltam que um repertório de HS pobre, principalmente em termos de empatia, expressão de sentimentos e resolução de problemas, parece estar associado a dificuldades interpessoais, envolvendo problemas de comportamento.

Não há consenso na literatura acerca da definição de problemas de comportamento. O presente estudo utilizará a definição funcional adotada por Bolsoni-Silva (2003), que define como problemas de comportamento déficits ou excessos comportamentais que prejudicam a interação da criança com pares e adultos de sua convivência, podendo ser classificados em externalizantes ou internalizantes.

Dentro da literatura de psicopatologia do desenvolvimento, Hinshaw (1992) e Pacheco, Alvarenga, Reppold, Piccinini e Hutz (2005) ressaltam que problemas de comportamento externalizantes (expressados predominantemente em relação a outras pessoas) são mais frequentes em transtornos que envolvem agressividade física ou verbal, comportamentos opositores ou desafiadores, condutas antissociais (mentir e roubar) e comportamentos de risco (como uso de substâncias psicoativas). Os problemas de comportamento internalizantes (expressados predominantemente em relação ao próprio indivíduo) são mais prontamente identificáveis em transtornos, como depressão, isolamento social, ansiedade e fobia social (Del Prette \& Del Prette, 2005; Gresham \& Elliott, 1990; Hinshaw, 1992; Patterson, Reid, \& Dishion, 2002). Entende-se que os problemas de comportamento concorrem com a saúde mental e desenvolvimento adequado de crianças e adolescentes e, por isso, consistem em um importante foco de estudo para a Psicologia (Orti, 2014).

Pesquisas apontam que, quanto maior a frequência de problemas de comportamento apresentados pelas crianças, menor o repertório de HS e o desempenho acadêmico das mesmas (Cia \& Barham, 2009; Cia, Pamplin, \& Del Prette, 2006; D’Avila-Bacarji, Marturano, \& Elias, 2005; Del Prette \& Del Prette, 2005). Nesse sentido, alguns autores consideram déficits no repertório de HS como fatores de risco para a realização acadêmica e para o desenvolvimento socioemocional (Del Prette \& Del Prette, 2005; Gresham, 2004) que podem se agravar na adolescência e idade adulta. Pode-se supor que esse risco seja ainda maior para crianças que apresentam comorbidade entre baixo desempenho escolar e problemas de comportamento, o que justifica considerar essas crianças como um grupo mais vulnerável.

Algumas pesquisas verificaram que a aquisição de HS implica na diminuição da ocorrência de comportamentos problemáticos em crianças. Baraldi e Silvares (2003) avaliaram o efeito do treinamento de HS e de resolução de problemas em crianças e observaram uma diminuição da ocorrência de comportamentos problemáticos, comparativamente a um grupo controle, e uma melhora da qualidade das suas relações familiares. Elias e Marturano (2004) encontraram resultados semelhantes, aplicando um programa para o desenvolvimento de HS com ênfase nas habilidades de solução de problemas interpessoais em crianças.

Baixo desempenho escolar é caracterizado como desempenho, em notas ou tarefas, abaixo de um nível esperado para a idade, habilidade e potencial de um indivíduo. É, portanto, uma discrepância entre a aptidão acadêmica e seu desempenho (Ellis \& Bernard, 2009). A maioria das crianças com baixo desempenho escolar é negativamente avaliada em sua competência social por colegas, professores e até mesmo por pais (Cia \& Barham, 2009; Del Prette \& Del Prette, 2005).

Estudos têm mostrado que crianças com baixo desempenho escolar podem apresentar problemas de comportamento, e vice-versa, caracterizando uma comorbidade frequente entre esses dois problemas (Bandeira, Rocha, Souza, Del Prette, \& Del Prette, 2006; Del Prette \& Del Prette, 2005). As evidências mostram que as crianças que não têm competência social e emocional desenvolvidas podem enfrentar um atraso na aquisição de habilidades acadêmicas fundamentais (Barreto, Freitas, \& Del Prette, 2011; Heydenberk \& 
Heydenberk, 2007). Além disso, as crianças que apresentam desempenho acadêmico abaixo do esperado numa fase tão precoce também correm o risco de exacerbação de comportamentos desadaptativos (McCabe \& Altamura, 2011).

Há evidência empírica de que os três domínios - acadêmico, interpessoal e comportamental - são interdependentes. Por exemplo, problemas de comportamento na meninice são preditores de resolução pobre das tarefas de desenvolvimento da fase, referentes ao desempenho escolar e ao relacionamento com os pares (Elias \& Marturano, 2014). Trajetórias de desenvolvimento com influências entre domínios foram identificadas para HS e desempenho acadêmico (Chen, Huang, Chang, Wang, \& Li, 2010), HS e problemas de comportamento (Burt \& Roisman, 2010; Van Lier \& Koot, 2010), desempenho e problemas de comportamento (Moilanen, Shaw, \& Maxwell, 2010).

A identificação de HS como um fator de proteção no curso do desenvolvimento humano tem estimulado intervenções para a aprendizagem dessas habilidades entre grupos e contextos distintos, com populações clínicas e não clínicas (Murta, 2005). A proposição de programas de HS na escola foi destacada por Gresham (2004) defendendo três vertentes de diferentes amplitudes, complementares e sequenciadas, na implantação desses programas na escola; os programas universais, para todas as crianças da escola, os seletivos, para crianças de risco em pequenos grupos que não se beneficiaram dos universais, e os individualizados, para atendimento das crianças que não respondem positivamente às duas estratégias anteriores.

Um programa de intervenção do tipo universal almejando aumentar as habilidades de solução de problemas interpessoais (HSPI) em crianças de ensino fundamental foi implementado e avaliado por Borges e Marturano (2003). Participaram 55 crianças da $1^{\mathrm{a}}$ série, designadas para a condição intervenção e condição comparação. Os resultados indicaram índices de HSPI superiores entre as crianças da condição intervenção em comparação às crianças da condição controle. As observações feitas ao longo do programa evidenciaram que os alunos que inicialmente se envolviam mais em conflitos diminuíram sua participação nesses eventos durante a intervenção.

Para estabelecer uma relação funcional entre HS e dificuldades na aprendizagem, Molina e Del Prette (2006) em estudo experimental com 16 estudantes entre sete e 13 anos que apresentavam dificuldades de leitura e escrita, verificaram que o grupo submetido ao programa de HS apresentou as melhoras esperadas no repertório social e ganhos acadêmicos, enquanto que o grupo submetido ao treinamento acadêmico melhorou o desempenho acadêmico, mas não obteve ganhos no repertório social. As autoras concluíram que os resultados favorecem a hipótese da relação funcional entre HS e o desempenho acadêmico.

Um aspecto a ser destacado no presente estudo consiste na relevância em investigar a relação entre HS, desempenho acadêmico e problemas de comportamento em crianças do $5^{\circ}$ ano do ensino fundamental. Achados na literatura (Borges \& Marturano, 2009; Borges \& Marturano, 2010; Cia \& Barham, 2009; Rodrigues, Dias \& Freitas, 2010) demonstram que extensos estudos foram publicados a fim de verificar a associação entre essas variáveis em crianças no início da escolarização. Portanto, são necessários estudos que explorem esses efeitos também em crianças que estão deixando as séries iniciais, no intuito de verificar os recursos desenvolvidos que atuam como protetivos ao desenvolvimento, principalmente ao pesar que a próxima etapa escolar contará com desafios mais complexos tanto no plano cognitivo como afetivo. Diante desses pressupostos, entende-se que compreender as relações entre HS, desempenho escolar e problemas de comportamento tem implicações diretas para o delineamento de programas de intervenção de caráter universal no contexto escolar que visem o desenvolvimento/aprimoramento de HS como fator de proteção ao desenvolvimento. Segundo Murta (2005), uma intervenção de caráter universal dirigida a indivíduos expostos a fatores de risco, mas ainda não acometidos por problemas interpessoais deve promover o incremento de suas HS, visto que atuam como fator de proteção e são concorrentes a problemas de comportamento.

Em relação aos estudos de prevenção universal que envolvem somente crianças, a literatura nacional apresenta pesquisas com objetivos mais focais e com um grande número de sessões (Elias, Marturano \& Motta-Oliveira, 2012; Vetorazzi et al., 2005). Assim, tornam-se necessários programas com um menor número de sessões, para que o uso possa ser viabilizado em contextos educacionais de forma a não atrapalhar ou concorrer com as demais atividades acadêmicas.

Com base nisso, este estudo teve por objetivo avaliar as HS, problemas de comportamento e desempenho acadêmico em crianças do $5^{\circ}$ ano do ensino fundamental I, antes e após um programa de intervenção de caráter universal para o desenvolvimento de HS em ambiente escolar. 


\section{Método}

\section{Participantes}

A amostra contou com 54 crianças, de ambos os sexos, regularmente matriculadas em uma escola pública municipal de ensino fundamental I de uma cidade de pequeno porte do interior de São Paulo e duas professoras. Foi realizado um sorteio randômico entre as classes de $5^{\circ}$ anos participantes de um projeto maior de treinamento de professores (ao qual este estudo está vinculado), a fim de constituir o grupo de intervenção (GI). Para o grupo de comparação (GC) foi solicitada a colaboração de professoras que não participavam do treinamento e também foi realizado um sorteio randômico entre as duas que se disponibilizaram. Os participantes de GC não receberam nenhum tipo de intervenção. Os responsáveis legais pela criança assinaram o Termo de Consentimento Livre e Esclarecido autorizando a participação no projeto. Como todos os pais assinaram, participaram todos os alunos de cada sala de aula sorteada.

O GI foi composto por 27 crianças, sendo 15 meninos e 12 meninas (média de idade $=9 \mathrm{a} 11 \mathrm{~m}$ ) e GC por 27 crianças, sendo 12 meninos e 15 meninas (média de idade $=10 \mathrm{a} 4 \mathrm{~m}$ ). Por se tratar de um programa de intervenção de caráter universal, não houve critério de seleção para inclusão ou exclusão dos participantes. Todas as crianças passaram por avaliações quanto às HS, características comportamentais e desempenho acadêmico antes e após a intervenção. A professora de GI participou da intervenção, realizando o treinamento na sala de aula pela qual era responsável durante quatro meses. A professora de GC não conduziu o treinamento, mas colaborou como informante para as avaliações de seus alunos.

\section{Instrumentos e Materiais}

- Teste de Desempenho Escolar (TDE): para obter um índice do desempenho escolar das crianças, foi utilizado o Teste de Desempenho Escolar (Stein, 1994), um instrumento com propriedades psicométricas adequadas (confiabilidade interna) que avalia as capacidades fundamentais para o desempenho escolar. Esse teste foi concebido para a avaliação de escolares de primeira a sexta séries do ensino fundamental e é composto por três subtestes: a) escrita, envolvendo a escrita do nome próprio e de 34 palavras isoladas, apresentadas sob a forma de ditado; b) aritmética, requerendo a solução oral de três problemas e cálculos de 35 operações aritméticas por escrito; e c) leitura, requerendo o conhecimento de setenta palavras isoladas do contexto.

- Social Skills Rating System (SSRS-BR) - Sistema de Avaliação de Habilidades Sociais (Bandeira, Del Prette, Del Prette, \& Magalhães, 2009). Trata-se de um conjunto de três escalas padronizadas (de HS, problemas de comportamento e desempenho acadêmico), que podem ser usadas com alunos de pré-escola e ensino fundamental. As escalas são apresentadas em três versões: autoavaliação do aluno, questionário para pais e para professores. Este estudo utilizou o SSRS versão para professores. Nessa versão, o professor avalia cada aluno em termos da frequência de emissão de HS, sendo 30 itens divididos em subescalas de: responsabilidade/cooperação (FR), asserção (FA), autocontrole (FT), autodefesa (FAD), cooperação com pares (FC); comportamentos problemáticos, sendo 18 itens, divididos em duas subescalas: comportamentos externalizantes (CPEX) e comportamentos internalizantes (CPINT); e competência acadêmica (nove itens), bem como a importância dos itens de HS. As escalas de frequência e importância são respondidas com base em três pontos (nunca, algumas vezes e muito frequentemente, no primeiro caso, e não importante, importante e crítico, no segundo). $\mathrm{Na}$ escala de competência acadêmica, o professor compara o aluno com as outras crianças da sala, utilizando uma escala de cinco pontos (entre os $10 \%$ piores, $20 \%$ piores, $40 \%$ médios, $20 \%$ bons ou entre os $10 \%$ ótimos) em leitura, matemática, motivação geral, participação dos pais, funcionamento intelectual e comportamento geral em classe.

- Programa Posso Pensar - PPP (Elias, 2012): programa de caráter universal que tem como objetivo desenvolver HS com foco nas habilidades de solução de problemas interpessoais. É constituído de 40 lições a serem trabalhadas de diferentes temas com complexidade crescente (inicia com o reconhecimento de sentimentos, até a escolha da melhor solução para determinado problema, verificando suas consequências). Cada lição possui um roteiro a ser seguido que fornece uma história a ser lida e trabalhada com as crianças por meio de atividades lúdicas propostas com possibilidades de ajustes às necessidades das turmas. No final de cada lição existe uma atividade de reforço a ser realizada no dia posterior ao da aplicação. Os roteiros das lições incluem objetivos/metas, uma lista dos recursos necessários para conduzi-la, esquemas de diálogos com as crianças e sugestões de jogos a serem desenvolvidos. As lições são agrupadas 
em duas grandes categorias: habilidades prévias para resolução de problemas e habilidades para resolução de problemas; em ambas as categorias são trabalhadas outras HS, como empatia, assertividade, autocontrole e cooperação, que são necessárias para o desenvolvimento das habilidades de solução de problemas, foco do programa. As habilidades prévias para resolução de problemas abrangem 15 lições, tendo como meta ensinar a: identificar os próprios sentimentos e os sentimentos dos outros, considerar o ponto de vista dos outros (diferentes sentimentos) e sequenciar os eventos. Já as habilidades para resolução de problemas são compostas por 25 lições subsequentes, tendo por meta ensinar a: pensar em mais de uma solução (diferentes caminhos para um mesmo objetivo), considerar as consequências (ações e sentimentos) e decidir que solução tomar. O tempo utilizado para a aplicação de cada lição varia entre 30 e 40 minutos. Recomenda-se que seja aplicado de duas a três vezes por semana.

\section{Procedimento}

Este estudo está ligado a um projeto maior que visa ao treinamento de professores para o desenvolvimento de HS das crianças em sala de aula. Para composição da amostra, inicialmente, foi realizado um sorteio randômico entre as classes de $5^{\circ}$ anos da escola das professoras que participavam do treino (projeto maior) e a outra entre as salas das professoras que não participavam do treino. Professoras e responsáveis legais das crianças assinaram os Termos de Consentimento Livre e Esclarecido destinados respectivamente para cada um em dias e horários previamente agendados na escola.

As avaliações diretas com as crianças quanto ao desempenho escolar, avaliado por meio do TDE, foram realizadas pelas pesquisadoras na biblioteca da escola durante o horário de aula, de forma que não atrapalhasse o conteúdo programático da classe e mediante consentimento da professora. As professoras avaliaram os alunos em relação às HS, problemas de comportamento e desempenho acadêmico respondendo ao SSRS fora do horário de sala de aula. Importante destacar que o SRRS avalia HS totais e subclasses de HS, que envolvem as habilidades de solução de problemas interpessoais.

Todas as avaliações ocorreram antes e após o treino em HS realizado pela professora em sala de aula. O programa Posso Pensar foi aplicado de duas a três vezes por semana, na sala de aula, durante o horário letivo (autorização prévia de Secretaria da Educação do município), sendo que a aplicação durou em média quatro meses. Para a execução das atividades do programa, foram utilizados materiais gráficos, livros infantis e jogos. A professora de GI foi responsável por conduzir e aplicar o treino em sua sala de aula, enquanto a professora de GC não aplicou o treino e colaborou apenas como informante nas avaliações. A professora que aplicou a intervenção recebeu treinamento e supervisão semanal de profissional de Psicologia quanto ao uso do programa para o desenvolvimento das $\mathrm{HS}$ em dias e horários marcados.

\section{Aspectos Éticos}

O projeto foi aprovado pelo Comitê de Ética em Pesquisa da Faculdade de Filosofia, Ciências e Letras de Ribeirão Preto (FFCLRP-USP), processo 580/2011.1.1426.59.4, e desenvolveu-se com autorização da Secretaria de Educação do município. Pais/ responsáveis e professores assinaram o Termo de Consentimento Livre e Esclarecido destinado a cada um. Para as crianças de GC, foi oferecido o treinamento em HS (por profissional de Psicologia) após a conclusão do estudo.

\section{Tratamento dos Dados}

Os dados obtidos por meio do SSRS (versão para professores) e TDE foram pontuados com base nos procedimentos indicados nos seus respectivos manuais. Os objetivos da análise estatística foram comparar os grupos comparação e intervenção em cada avaliação e contraposto os diferentes momentos de avaliação em cada grupo estudado, sendo que serão apresentadas, neste estudo, apenas as comparações que foram significativas. Inicialmente foi realizada uma análise exploratória de dados por meio de medidas de resumo. As comparações foram feitas por meio de contrates ortogonais utilizando o modelo linear de efeitos mistos (efeitos aleatórios e fixos). Esse modelo é utilizado na análise de dados onde as respostas de um mesmo indivíduo estão agrupadas e a suposição de independência entre as observações em um mesmo grupo não é adequada (Schall, 1991). Para a utilização desse modelo, é preciso que seus resíduos tenham distribuição normal com média zero e variância constante. Como esse pressuposto não foi atendido, realizou-se uma transformação logarítmica na variável resposta. O ajuste do modelo foi feito por meio do procedimento PROC MIXED do software SAS $^{\circledR} 9.1$ (2003). 


\section{Resultados}

A Tabela 1 apresenta as médias e valores de $p$ e $t$ para as escalas de competência acadêmica (CA), habilidades sociais (HS) e subescalas de responsabilidade/ cooperação (FR), asserção (FA), autocontrole (FT), autodefesa (FAD), cooperação com pares (FC), escala de total de comportamentos problemáticos (TT-CP) e subescalas de comportamentos externalizantes (CPEX) e comportamentos internalizantes (CPINT) em relação aos grupos estudados entre as fases de avaliação inicial e pós-intervenção ou espera, a partir dos dados obtidos por meio das análises do SSRS.

De acordo com os dados apresentados na escala de competência acadêmica, é possível perceber que GI aumentou sua média pós-intervenção, sendo essa diferença significativa. Quanto a GC também apresentou médias superiores na avaliação pós-espera, sendo significativa. Foram realizadas comparações entre os grupos GI e GC nos diferentes momentos e não existiram diferenças significativas.

No que se refere à escala de habilidades sociais, observa-se que GI apresentou diferença significativa entre as fases pré e pós-intervenção. Quanto a GC, não foram observadas diferenças significativas entre as avaliações. Nas comparações entre os grupos, não foram observadas diferenças significativas na avaliação inicial, no entanto, na avaliação pós-intervenção ou espera, houve diferença (estimativa da diferença entre as médias $=-10,34 ; t=-2,62 ; p<0,01)$, a favor de GI.

Nas subescalas de HS (responsabilidade/cooperação - FR, asserção - FA, autocontrole - FT,

Tabela 1

Médias e Valores de te $p$ em Diferentes Grupos e Momentos Avaliados Pelo SSRS

\begin{tabular}{|c|c|c|c|c|c|}
\hline Variável & Grupo & $\begin{array}{c}\text { Pré-intervenção } \\
\text { Média }(D P)\end{array}$ & $\begin{array}{c}\text { Pós-intervenção } \\
\text { Média }(D P)\end{array}$ & $t_{\text {pré-pós }}$ & $P_{\text {pré-pós }}$ \\
\hline \multirow[t]{2}{*}{ CA } & GI & $24,93(14,22)$ & $32,56(9,25)$ & $-2,56$ & $0,012^{* *}$ \\
\hline & GC & $29,04(12,47)$ & $30,3(12,41)$ & $-2,03$ & $0,04 *$ \\
\hline \multirow[t]{2}{*}{ TT-HS } & GI & $37,15(13,22)$ & $47,37(12,95)$ & $-6,1$ & $<0,001 * *$ \\
\hline & GC & $34,78(17,15)$ & $35,78(16,32)$ & $-1,41$ & 0,164 \\
\hline \multirow[t]{2}{*}{ TT-FR } & GI & $20,33(6,67)$ & $23,93(6,57)$ & $-3,6$ & $0,006^{* *}$ \\
\hline & GC & $18,3(9,56)$ & $19,89(9,05)$ & $-3,25$ & $0,001 * *$ \\
\hline \multirow[t]{2}{*}{ TT-FA } & GI & $10,78(3,51)$ & $13,96(3,8)$ & $-5,37$ & $<0,001 * *$ \\
\hline & GC & $9,85(6,48)$ & $9,63(4,68)$ & $-2,01$ & $0,048^{*}$ \\
\hline \multirow[t]{2}{*}{ TT-FT } & GI & $11,59(4,16)$ & $14,19(4,07)$ & $-5,63$ & $<0,001 * *$ \\
\hline & GC & $9,74(4,48)$ & $10,15(4,63)$ & $-1,09$ & 0,279 \\
\hline \multirow[t]{2}{*}{ T'T-FAD } & GI & $3,15(1,38)$ & $4,85(1,35)$ & $-6,18$ & $<0,001 * *$ \\
\hline & GC & $3(4,54)$ & $2,7(1,94)$ & $-1,67$ & 0,099 \\
\hline \multirow[t]{2}{*}{ TT-FC } & GI & $4,56(1,83)$ & $5,89(1,67)$ & $-5,66$ & $<0,001 * *$ \\
\hline & GC & $3,89(2,01)$ & $4,44(2,03)$ & $-2,83$ & $0,005^{* *}$ \\
\hline \multirow[t]{2}{*}{ TT-CP } & GI & $8,74(8,96)$ & $7,3(6,75)$ & 2,62 & $0,011 * *$ \\
\hline & GC & $4,15(3,66)$ & $4,07(3,97)$ & 0,83 & 0,407 \\
\hline \multirow[t]{2}{*}{ CPEX } & GI & $5,67(7,13)$ & $4,33(5,42)$ & 2,18 & $0,034 * *$ \\
\hline & GC & $2,33(2,45)$ & $2,59(2,82)$ & 0,21 & 0,834 \\
\hline \multirow[t]{2}{*}{ CPINT } & GI & $3,52(3,8)$ & $3,3(2,07)$ & 0,91 & 0,367 \\
\hline & GC & $1,96(2,08)$ & $1,74(1,81)$ & 0,94 & 0,348 \\
\hline
\end{tabular}

Nota ${ }^{1}$. Pré-intervenção: $n=27$; pós-intervenção: $n=27$. * $p<0,05 ; * * p<0,01$.

Nota 2 . CA $=$ Competência acadêmica, $\mathrm{HS}=$ Habilidades sociais, $\mathrm{FR}=$ Responsabilidade $/$ cooperação, FA $=$ Asserção, FT $=$ Autocontrole, $\mathrm{FAD}$ $=$ Autodefesa, $\mathrm{FC}=$ Cooperação com pares, $\mathrm{T}$ T-CP $=$ Total de comportamentos problemáticos, $\mathrm{CPEX}=$ Comportamentos externalizantes e CPINT $=$ Comportamentos internalizantes. 
autodefesa - FAD e cooperação com pares - FC), GI apresentou diferença significativa entre as avaliações pré e pós-intervenção em todas subescalas. Quanto a GC apresentou diferença estatisticamente significativa entre as avaliações inicial e pós-intervenção nas subescalas de responsabilidade/cooperação e cooperação com pares. Quando comparados GI e GC, não foram observadas diferenças significativas na avaliação inicial, já, na avaliação pós-intervenção ou espera, GI apresentou médias superiores e estatisticamente significativas em relação à GC em diferentes subescalas (FA - estimativa da diferença entre as médias $=-0,283 ; t=-2,39$; $p<0,019$; FT - estimativa da diferença entre as médias $=-3,9 ; t=-3,3 ; p<0,001 ;$ FAD - estimativa da diferença entre as médias $=-0,453 ; t=-3,12 ; p<0,002$; $\mathrm{FC}-$ estimativa da diferença entre as médias $=-1,3 ; t$ $=-2,58 ; p<0,011)$.

Com relação à escala de comportamentos problemáticos, observa-se que GI apresentou melhoras significativas entre as avaliações pré e pós-intervenção. Com relação à GC, não apresentou diferenças significativas entre as avaliações. Nas comparações entre os grupos, foi observada diferença significativa na avaliação inicial, onde GI apresentava maiores resultados, ou seja, maiores problemas de comportamento (estimativa da diferença entre as médias $=-0,69 ; t=-2,62$; $p<0,011)$, no entanto, na avaliação pós-intervenção, não houve essa diferença em relação à subescala de comportamentos problemáticos.

$\mathrm{Na}$ subescala de comportamentos externalizantes, GI apresentou diferença significativa entre as médias na avaliação inicial e pós-intervenção. Quanto a GC, esse grupo não apresentou diferenças significativas entre as avaliações. Nas comparações entre os grupos, foi observada diferença significativa na avaliação inicial (estimativa da diferença entre as médias $=-0,669 ; t=$ $-2,19 ; p<0,034)$, onde GI apresentava mais problemas; não foram observadas diferenças após a intervenção.

No que tange à subescala de comportamentos internalizantes, percebe-se que embora GI e GC tenham apresentado diferença nas médias entre as avaliações, essa não foi estatisticamente significativa para ambos os grupos. Nas comparações entre os grupos, foi observada diferença significativa nos dois momentos de avaliação, onde GI apresentou mais problemas (estimativa da diferença entre as médias na avaliação inicial $=-0,427 ; t=-2,03 ; p<0,046$; e na avaliação pós-intervenção $=-0,447 ; t=-2,15 ; p<0,035)$.

A Tabela 2 apresenta médias e valores de $p$ e $t$ nas comparações em relação aos grupos estudados entre as fases de avaliação inicial e pós-intervenção ou espera, a partir dos dados obtidos por meio das análises do TDE para as variáveis de escrita $(\mathrm{E})$, aritmética $(\mathrm{A})$, leitura $(\mathrm{L})$ e escore total bruto (TT-EBT).

Em relação ao desempenho acadêmico avaliado pelo TDE, é possível dizer que GI apresentou médias superiores em escrita após a intervenção, sendo essa diferença significativa. O mesmo foi observado em GC. Nas comparações entre os grupos em relação à variável escrita, foi observada diferença significativa na avaliação inicial, (estimativa da diferença entre as médias $=-0,369$; $t=-1,94 ; p<0,055)$, e na avaliação pós-intervenção

Tabela 2

Médias e Valores de te p em Diferentes Grupos e Momentos Avaliados Pelo TDE

\begin{tabular}{lccccc}
\hline Variável & Grupo & $\begin{array}{c}\text { Pré-intervenção } \\
\text { Média }(D P)\end{array}$ & $\begin{array}{c}\text { Pós-intervenção } \\
\text { Média }(D P)\end{array}$ & $t_{\text {pré-pós }}$ & $p_{\text {pré-pós }}$ \\
\hline E & GI & $26,56(3,62)$ & $28,11(4,13)$ & $-3,54$ & $0,007^{* *}$ \\
& GC & $22,89(8,43)$ & $23,59(8,36)$ & $-1,95$ & $0,054^{*}$ \\
A & GI & $18,15(3,22)$ & $18,74(3,45)$ & $-1,14$ & 0,256 \\
& GC & $18,44(4,81)$ & $18,37(4,77)$ & 0,24 & 0,812 \\
L & GI & $64,89(4,82)$ & $67,19(3,45)$ & $-2,88$ & $0,005^{* *}$ \\
\multirow{2}{*}{ TT-EBT } & GC & $62,89(11,56)$ & $63,15(11,72)$ & $-0,44$ & 0,663 \\
& GI & $109,81(9,15)$ & $114,04(9,39)$ & $-3,43$ & $0,001^{* *}$ \\
& GC & $104,22(22,67)$ & $105,26(22,7)$ & $-0,81$ & 0,422 \\
\hline
\end{tabular}

Nota . Pré-intervenção: $n=27$; pós-intervenção: $n=27 . * p<0,05 ; * * p<0,01$.

Nota $2 . \mathrm{E}=$ Escrita, $\mathrm{A}=$ Aritmética, $\mathrm{L}=$ Leitura e TT-EBT $=$ Escore total bruto

Psico-USF, Bragança Paulista, v. 21, n. 1, p. 49-61, jan./abr. 2016 
ou espera (estimativa da diferença entre as médias = $-4,261 ; t=-2,46 ; p<0,016)$, a favor de GI.

Com relação à variável aritmética, tanto GI como GC não apresentaram diferenças significativas entre as avaliações. Nas comparações entre os grupos GI e GC também não foram observadas diferenças significativas.

$\mathrm{Na}$ variável leitura, nota-se que GI apresentou diferença significativa entre as avaliações inicial e pós-intervenção. Quanto a GC, embora tenha apresentado aumento na média entre os dois momentos de avaliação, essa diferença não foi significativa. Quando comparados GI e GC, não foram observadas diferenças significativas entre as médias na avaliação inicial, já na avaliação pós-intervenção ou espera, GI apresentou médias superiores e marginalmente significativas (estimativa da diferença entre as médias $=-3,874 ; t=-1,67$; $p<0,099)$.

Finalmente no escore total bruto, GI apresentou diferença significativa entre as médias das avaliações inicial e pós-intervenção. Quanto a GC, ao comparar as avaliações pré e pós-espera, não foram observadas diferenças significativas. Nas comparações entre os grupos GI e GC, na avaliação inicial, embora GI apresentasse média superior, essa diferença não foi significativa; na avaliação pós-intervenção ou espera, GI apresentou médias novamente superiores e marginalmente significativas (estimativa da diferença entre as médias $=-8,663 ; t=-1,84 ; p<0,07)$.

\section{Discussão}

Tendo como objetivo avaliar as HS, problemas de comportamento e desempenho acadêmico em crianças do $5^{\circ}$ ano do ensino fundamental I antes e após treino específico para o desenvolvimento das HS em ambiente acadêmico, os resultados obtidos foram significativos, apontando que programas de caráter universal no contexto escolar constituem uma alternativa possível e encorajadora. A literatura vem apontando para o contexto escolar como uma possibilidade de atuação em grande escala (Gresham, 2004; Borges \& Marturano, 2003; Caballo, 2010), ressaltando a importância das intervenções de caráter universal em HS, pois atuam como fator de proteção ao desenvolvimento (Murta, 2005).

Os dados obtidos por meio do SSRS sugeriram resultados importantes. No que tange às HS, GI apresentou diferença significativa entre as avaliações inicial e pós-intervenção, o que não ocorreu no GC. Observou-se ainda que em todas as subescalas de HS (responsabilidade/cooperação, asserção, autocontrole, autodefesa e cooperação com pares), GI apresentou diferença significativa entre as avaliações inicial e pós-intervenção; GC apresentou diferença estatisticamente significativa entre as avaliações inicial e pós-espera nas subescalas de responsabilidade/ cooperação e cooperação com pares (habilidades que rotineiramente são trabalhadas pelas professoras, visto que são centrais na relação ensino-aprendizagem). Os resultados obtidos por GI vão ao encontro da literatura de que crianças que passam por treino em HS se beneficiam na aquisição dessas habilidades (Elias et al., 2012; Gresham, 2004).

Com relação aos comportamentos problemáticos, notou-se que os participantes de GI apresentaram melhoras significativas entre as avaliações inicial e pós-intervenção, no que se refere ao total de problemas de comportamento e em problemas de comportamento externalizantes. O mesmo não ocorreu com GC nos dois momentos de avaliação; não foram observadas diferenças significativas, tanto no total de problemas de comportamento como em comportamentos externalizantes, ou seja, os problemas permaneceram no mesmo nível que o inicial. Esses resultados corroboram os resultados encontrados na literatura de que a aquisição de HS promove a diminuição da ocorrência de comportamentos problemáticos, visto que leva as crianças a pensarem em seus problemas e nas consequências possíveis às suas resoluções (Elias \& Marturano, 2004; Baraldi \& Silvares, 2003). Segundo Barker et al. (2010), a aquisição de HS na infância pode ser vista com uma forma de impedir ou controlar o comportamento agressivo.

Quanto aos problemas de comportamento internalizantes, observou-se que tanto GI como GC não apresentaram diferenças significativas entre as avaliações. Esse dado está em consonância com os achados da literatura, que aponta que os problemas internalizantes frequentemente não são reconhecidos pelo ambiente, especialmente em razão dos sintomas serem caracterizados pelo excesso de autocontrole da criança (Merrell, 2008). Nesse sentido, raramente são alvo de queixa por parte de pais e professores e poucas pesquisas dedicam-se a identificar variáveis específicas com relação à internalização e intervenções que possam ser eficazes (Orti, 2014). Além disso, crianças com problemas de comportamento internalizantes (por exemplo, aquelas que não fazem perguntas) acabam recebendo menos atenção do que crianças com problemas de comportamento externalizantes (por exemplo, aquelas que gritam). 
Assim, de acordo com Cia e Barham (2009), as avaliações de problemas internalizantes podem ser menos acuradas do que as avaliações de comportamentos externalizantes, como refletido nas correlações sistematicamente menores envolvendo os comportamentos internalizantes do que as envolvendo os comportamentos externalizantes. Ainda com relação aos comportamentos internalizantes, é possível pensar que algumas características do programa utilizado (trabalho enfático com a construção de vocabulário em HS, utilizando verbalização de vivências, sentimentos e pensamentos) podem não favorecer as crianças mais quietas e menos falantes, que é uma característica das crianças mais internalizantes.

Os resultados relacionados ao desempenho escolar apontam para uma discrepância entre a avaliação realizada pelas professoras e a avaliação direta com as crianças, feita pelas pesquisadoras. As professoras pareceram superestimar a produção de seus alunos (principalmente as do GC, onde os avanços acadêmicos foram discretos). Por meio da escala de competência acadêmica (avaliada pelas professoras), tanto os participantes de GI como GC apresentaram progressos significativos no desempenho escolar entre as avaliações inicial e pós-intervenção ou espera. $\mathrm{Na}$ comparação entre os grupos, não houve diferença nos diferentes momentos de avaliação, ou seja, segundo as professoras, as crianças de ambos os grupos melhoraram no decorrer do tempo.

Já ao verificar o desempenho escolar por meio da avaliação direta com as crianças (utilizando o TDE), observou-se que GI apresentou progressos significativos no escore total, em escrita e leitura entre as avaliações inicial e pós-intervenção. Com relação à GC apenas foram observadas diferenças significativas entre as avaliações na variável escrita. $\mathrm{Na}$ variável aritmética, tanto GI como GC não apresentaram diferença significativa nas médias das diferentes avaliações.

Em relação às aquisições em aritmética, pode-se inferir que não houve diferença significativa por se tratar de uma característica no currículo escolar das salas investigadas, onde as atividades relacionadas à aritmética são mais trabalhadas a partir do segundo semestre, e as avaliações foram realizadas no primeiro semestre. Outra possibilidade é a de que o programa aplicado, por constituir atividades predominantemente verbais e que podem envolver atividades de leitura e escrita dependendo do ano escolar aplicado, pode favorecer menos atividades de aritmética e mais de leitura e escrita. Vale ressaltar que o objetivo do programa é desenvolver HS, como consequência, diminuir problemas de comportamento e aumentar a capacidade de atenção ao conteúdo acadêmico.

De forma geral, ao comparar GI e GC quanto ao desempenho acadêmico, os resultados vão ao encontro do proposto pela literatura, considerando que o diferencial do treino de HS não está somente em promover ou aprimorar o repertório de HS nas crianças, mas também em gerar impacto positivo no desempenho escolar, tal como foi verificado por diferentes trabalhos (Del Prette \& Del Prette, 2005; Elias \& Marturano, 2004; Molina \& Del Prette, 2006). Acredita-se que o treino em HS gera nas crianças maior capacidade de atenção, reflexão, empatia, cooperação e resolução de problemas, o que potencializa um aprendizado escolar mais efetivo.

Ao comparar os resultados das avaliações antes e após o programa de intervenção e obtido um aumento estatisticamente significativo em grande parte das variáveis estudadas, pode-se dizer que o programa de intervenção foi eficaz para as crianças desse agrupamento sociocultural, no sentido de melhorar/promover HS, competências acadêmicas e de atenuar problemas de comportamento.

Nesse sentido, Marturano (2004) evidenciou em seus estudos que as crianças que passaram por programas que ajudavam a superar tanto as dificuldades escolares quanto as interpessoais apresentaram ganhos mais persistentes no progresso escolar e na redução das dificuldades emocionais, quando comparadas com crianças que tiveram apenas atendimento para superar as dificuldades acadêmicas. Assim, o treinamento em HS no âmbito escolar como parte das atividades curriculares contribui não só para melhorar a qualidade das HS das crianças e reduzir os problemas de comportamento, como também para um melhor aproveitamento dos conteúdos escolares (Borges \& Marturano, 2010).

Em síntese, os dados obtidos pelo presente estudo pretendem contribuir, dentro de seus limites, para favorecer o planejamento de práticas mais assertivas de promoção de HS no contexto escolar. Importante destacar que o envolvimento da instituição escolar e das professoras participantes do estudo foi grande, mas também os resultados foram bastante positivos e reconhecidos por elas.

\section{Considerações Finais}

Para finalizar, algo deve ser dito sobre limitações metodológicas do estudo. O número de participantes e a restrição em apenas um determinado ano do ensino 
fundamental pode se configurar em um fator limitante para a generalização dos efeitos do programa. Para futuros estudos, a inclusão de um número maior de participantes, englobando diferentes anos do ensino fundamental, assim como a participação de múltiplos informantes que convivem com a criança em diferentes contextos poderiam trazer dados adicionais relevantes para as questões aqui tratadas.

De modo geral, os resultados produzidos pelos programas de intervenção desenvolvidos no cenário brasileiro e internacional têm apontado o amplo espectro de efeitos positivos que o treinamento em HS pode promover no desenvolvimento infantil. Por fim, os resultados deste estudo apontam para a importância de elaborar e implantar ações de caráter universal, visando ao aumento e à promoção do repertório de HS e à diminuição de problemas de comportamento no contexto escolar.

\section{Referências}

Bandeira, M., Del Prette, Z. A. P., Del Prette, A., \& Magalhães, T. (2009). Validação das escalas de habilidades sociais, comportamentos problemáticos e competência acadêmica (SSRS-BR) para o ensino fundamental. Psicologia: Teoria e Pesquisa, 25, 271282. Recuperado de http://www.scielo.br/pdf/ ptp/v25n2/a16v25n2.pdf

Bandeira, M., Rocha, S. S., Souza, T. M. P., Del Prette, Z. A. P., \& Del Prette, A. (2006). Comportamentos problemáticos em estudantes do ensino fundamental: Características da ocorrência e relação com habilidades sociais e dificuldade de aprendizagem. Estudos de Psicologia (Natal), 11, 199-208. doi: 10.1590/S1413-294X2006000200009

Baraldi, D., \& Silvares, E. (2003). Treino de habilidades sociais em grupo com crianças agressivas, associado à orientação dos pais: Análise empírica de uma proposta de atendimento. Em A. Del Prette \& Z. A. P. Del Prette (Eds.). Habilidades sociais, desenvolvimento e aprendizagem (pp. 235-258). Campinas, SP: Alínea.

Barker, E. D., Vitaro, F., Lacourse, E., Fontaine, N. M. G., Carbonneau, R., \& Tremblay, R. E. (2010). Testing the developmental distinctiveness of male proactive and reactive aggression with a nested longitudinal experimental intervention. Aggressive Behavior, 36, 127-140. doi: 10.1002/ab.20337.
Barreto, S. O., Freitas, L. C., \& Del Prette, Z. A. P. (2011). Habilidades sociais na comorbidade entre dificuldades de aprendizagem e problemas de comportamento: Uma avaliação multimodal. Psico, 42, 503-510. Recuperado de http://revistaseletronicas.pucrs.br/ojs/index.php/revistapsico/ article/view/7593

Bolsoni-Silva, A. T. (2003). Habilidades sociais educativas, variáveis contextuais e problemas de comportamento: Comparando pais e mães de pré-escolares (Tese de doutorado não publicada). Universidade de São Paulo, Ribeirão Preto, SP.

Bolsoni-Silva, A. T., \& Carrara, K. (2010). Habilidades sociais e análise do comportamento: Compatibilidades e dimensões conceitual-metodológicas. Psicologia em Revista (Belo Horizonte), 16(2), 330-350. Recuperado de http://pepsic.bvsalud. org/pdf/per/v16n2/v16n2a07.pdf

Borges, D., \& Marturano, E. (2003). Desenvolvendo habilidades de solução de problemas interpessoais no ensino fundamental. Paidéia, 12(24), 185-193. Recuperado de http://www.scielo.br/pdf/paideia/v12n24/07.pdf

Borges, D., \& Marturano, E. (2009). Aprendendo a gerenciar conflitos: Um programa de intervenção para a $1^{a}$ série do ensino fundamental. Paideia, 19(42), 17-26. doi: 10.1590/S0103-863X2009000100004

Borges, D., \& Marturano, E. (2010). Melhorando a convivência em sala de aula: Responsabilidades compartilhadas. Temas em Psicologia, 18, 123-136. Recuperado de http://pepsic.bvsalud.org/pdf/ tp/v18n1/v18n1a11.pdf

Burt, K. B., \& Roisman, G. I. (2010). Competence and psychopathology: Cascade effects in the NICHD Study of Early Child Care and Youth Development. Development and Psychopathology, 22, 557-567. doi: 10.1017/S0954579410000271.

Caballo, V. (1996). O treinamento em habilidades sociais. Em V. E. Caballo (Ed.), Manual de técnicas de terapia e modificação do comportamento (pp.181-309). São Paulo, SP: Ed. Santos.

Caballo, V. (2003). Manual de avaliação e treinamento das habilidades sociais. São Paulo, SP: Ed. Santos.

Caballo, V. (2010). Manual de avaliação e treinamento das habilidades sociais (S. M. Dolinsky, Trad.). São Paulo, SP: Ed. Santos. 
Chen, X., Huang, X., Chang, L., Wang, L., \& Li, D. (2010). Aggression, social competence, and academic achievement in Chinese children: A 5-year longitudinal study. Development and Psychopathology, 22, 583-592. doi: 10.1017/S0954579410000295.

Cia, F., \& Barham, E. J. (2009). Repertório de habilidades sociais, problemas de comportamento, autoconceito e desempenho acadêmico de crianças no início da escolarização. Estudos de Psicologia (Campinas), 26, 45-55. Recuperado de http://www. scielo.br/pdf/estpsi/v26n1/a05v26n1.pdf

Cia, F., Pamplin, R. C. O., \& Del Prette, Z. A. P. (2006). Comunicação e participação pais-filhos: Correlação com habilidades sociais e problemas de comportamento dos filhos. Paideia (Ribeirão Preto), 16, 395-408. doi: 10.1590/S0103-863X2006000300010

D’Avila-Bacarji, K. M. G., Marturano, E. M., \& Elias, L. C. S. (2005). Suporte parental: Um estudo sobre crianças com queixas escolares. Psicologia em Estudo, 10, 110-115. doi: 10.1590/ S1413-73722005000100013

Del Prette, A., \& Del Prette, Z. (2005). Psicologia das habilidades sociais na infância: Teoria e prática. Petrópolis, RJ: Vozes.

Del Prette, Z., \& Del Prette, A. (2003). Habilidades sociais e dificuldades de aprendizagem: Teoria e pesquisa sob um enfoque multimodal. In A. Del Prette \& Z. A. P. Del Prette (Eds.), Habilidades sociais, desenvolvimento e aprendizagem (pp. 167-206). Campinas, SP: Alínea.

Elias, L. C. S. (2012). Programa Posso Pensar - Desenvolvendo habilidades de solução de problemas interpessoais em escolares (Documento não publicado). Faculdade de Filosofia, Ciências e Letras de Ribeirão Preto, Universidade de São Paulo, Ribeirão Preto, SP.

Elias, L. C. S., \& Marturano, E. M. (2004). Habilidades de solução de problemas interpessoais e a prevenção dos problemas de comportamento em escolares. In E. M. Marturano, M. B. M. Linhares \& S. R. Loureiro (Eds.), Vulnerabilidade e proteção: Indicadores na trajetória de desenvolvimento do escolar (pp. 197-216). São Paulo, SP: Casa do Psicólogo.

Elias, L. C. S., \& Marturano, E. M. (2014). "Eu posso resolver problemas" e oficinas de linguagem: Intervenções para queixa escolar. Psicologia: Teoria e Pesquisa, 30, 35-44. doi: 10.1590/ S0102-37722014000100005
Elias, L. C. S., Marturano, E. M., \& Motta-Oliveira, A. M. A. (2012). Eu posso resolver problemas: Um programa para o desenvolvimento de habilidades de solução de problemas interpessoais. Temas em Psicologia, 20, 521-535. Recuperado de http:/ / www.temasempsicologia.org/arquivo/ download?ID_ARQUIVO=17

Ellis, A., \& Bernard, M. E. (2006). Rational emotive behavioral approaches to childhood disorders: Theory, practice and research. New York, NY: Springer Science.

Falcão, A. P. (2014). Avaliação da eficácia de uma intervenção em grupo com escolares para a promoção de melhores interaçôes sociais (Dissertação de mestrado não publicada). Universidade Estadual Paulista, Bauru, SP.

Falcone, E. O. (2002). Contribuições para o treinamento de habilidades de interação. In H. J. Guilhardi, M. B. B. P. Madi, P. P. Queiroz, \& M. C. Scoz (Eds.), Sobre comportamento e cognição. Contribuições para a construção da teoria do comportamento (pp. 91-104). Santo André, SP: ESETec.

Gresham, F. M. (2004). Current status and future directions of school-based behavioral interventions. School Psychology Review, 33, 326-343. Recuperado de http:/ / eric.ed.gov/ ?id=EJ683538

Gresham, F. M., \& Elliott, S. N. (1990). Social skills rating system: Manual. Circle Pines, MN: American Guidance Service.

Heydenberk, W., \& Heydenberk, R. (2007). More than manners: Conflict resolution in primary level classrooms. Early Childhood Education Journal, 35, 119-126. Recuperado de http://link.springer. com/article/10.1007/s10643-007-0185-4

Hinshaw, S. (1992). Externalizing behavior problems and academic underachievement in childhood and adolescence: Causal relationships and underlying mechanisms. Psychological Bulletin, 111, 127-155. Recuperado de http://www.ncbi.nlm.nih.gov/ pubmed/1539086

Marturano, E. M. (2004). Fatores de risco e proteção no desenvolvimento socioemocional de crianças com dificuldades de aprendizagem. In E. G. Mendes, M. A. Almeida \& L. C. A. Williams (Eds.), Avanços recentes em educação especial (pp. 159-165). São Carlos, SP: EDUFSCar.

McCabe, P. C., \& Altamura, M. (2011). Empirically valid strategies to improve social and emotional 
competence of preschool children. Psychology in the Schools, 48, 513-540. doi: 10.1002/pits.20570

Merrell, K. W. (2008). Helping students overcome depression and anxiety: A practical guide. New York, NY: Guilford.

Moilanen, K. L., Shaw, D. S., \& Maxwell, K. L. (2010). Developmental cascades: Externalizing, internalizing, and academic competence from middle childhood to early adolescence. Development and Psychopathology, 22, 635-653. doi: 10.1017/S0954579410000337.

Molina, R., \& Del Prette, Z. (2006). Funcionalidade da relação entre habilidades sociais e dificuldades de aprendizagem. Psico-USF, 11, 53-63. doi: 10.1590/ S1413-82712006000100007

Murta, S. G. (2005). Aplicações do treinamento em habilidades sociais: Análise da produção nacional. Psicologia: Reflexão e Crítica, 18, 283-291. Recuperado de http://www.scielo.br/pdf/prc/ v18n2/27480.pdf

Orti, N. P. (2014). Avaliação dos efeitos e variáveis do processo de intervenção com mães de crianças com problemas internalizantes (Dissertação de mestrado não publicada). Universidade Estadual Paulista. Bauru, SP.

Pacheco, J., Alvarenga, P., Reppold, C., Piccinini, C., \& Hutz, C. (2005). Estabilidade do comportamento antissocial na transição da infância para a adolescência: Uma perspectiva desenvolvimentista. Psicologia: Reflexão e Crítica, 18, 55-61. doi: 10.1590/ S0102-79722005000100008

Patterson, G., Reid, J., \& Dishion, T. (2002). Antisocial boys: Comportamento antissocial. Santo André, SP: Esetec.
Rodrigues, M., Dias, J., \& Freitas, M. (2010). Resolução de problemas interpessoais: Promovendo o desenvolvimento sociocognitivo na escola. Psicologia em Estudo (Maringá), 15(4), 831-839. Recuperado de Recuperado de http://www.redalyc.org/articulo. oa?id $=287123084019$

SAS Institute Inc. (2003). SAS/STAT® user's guide, version 9. Cary, NC: SAS Institute.

Schall, R. (1991). Estimation in generalized linear models with random effects. Biometrika, 78, 719-727. Recuperado de http://www.jstor.org/ stable $/ 2336923$ ?seq=1\#page_scan_tab_contents

Stein, L. M. (1994). TDE - Teste de desempenho escolar: Manual para aplicaşão e interpretação. São Paulo, SP: Casa do Psicólogo.

Van Lier, P. A. C., \& Koot, H. M. (2010). Developmental cascades of peer relations and symptoms of externalizing and internalizing problems from kindergarten to fourth-grade elementary school. Development and Psychopathology, 22, 569-582. doi: 10.1017/S0954579410000283.

Vettorazzi, A., Frare, E., De Souza, F. C., De Queiroz, F. P., De Luca, G. G., Moskorz, L., \& Kubo, O. M. (2005). Avaliação de um programa para ensinar comportamento empático para crianças em contexto clínico. Interação em Psicologia, 9(2), 355-369. Recuperado de http://ojs.c3sl.ufpr.br/ojs/index. php/psicologia/article/viewFile/4780/3668

Recebido em: 27/11/2014 Primeira reformulação em: 27/02/2015 Segunda reformulação em: 02/04/2015

Aprovado em: 09/04/2015 
Nota das autoras:

Apoio Financeiro: FAPESP

Sobre as autoras:

Luciana Carla dos Santos Elias é psicóloga, mestre em Saúde Mental pela Faculdade de Medicina de Ribeirão Preto-USP e doutora em Psicologia pela FFCLRP-USP. Atualmente é docente do Departamento de Psicologia da FFCLRP, ministra disciplinas de Psicologia da Educação e Educação e Subjetividade e orienta estágios em Psicologia Escolar e atendimento às dificuldades na aprendizagem e desenvolve pesquisas em habilidades sociais.

E-mail:lucaelias@ffclrp.usp.br

Márcia Viana Amaral é graduanda em Psicologia pela FFCLRP-USP, foi bolsista de iniciação científica com financiamento da Fundação de Amparo à Pesquisa do Estado de São Paulo (FAPESP), sob orientação da primeira autora, e, atualmente, desenvolve pesquisas na área de habilidades sociais.

E-mail:marcia_amarall@hotmail.com

Contato com as autoras:

Luciana Carla dos Santos Elias

Avenida Bandeirantes, 3900, Monte Alegre, Ribeirão Preto - SP

CEP: $14040-901$ 
